Article

\title{
Power Scalable Bi-Directional DC-DC Conversion Solutions for Future Aircraft Applications
}

\author{
Antonio Lamantia ${ }^{1}$, Francesco Giuliani ${ }^{1}$ and Alberto Castellazzi ${ }^{2, *}$ \\ 1 Blu Electronic Srl, Via Lavoratori Autobianchi 1, 20033 Desio (MB), Italy; \\ antonio.lamantia@bluelectronic.it (A.L.); fgiuliani121@gmail.com (F.G.) \\ 2 Solid-State Power Processing (SP2) Lab, Faculty of Engineering, Kyoto University of Advanced Science, \\ Kyoto 615-8577, Japan \\ * $\quad$ Correspondence: alberto.castellazzi@kuas.ac.jp; Tel.: +81-75-496-6504
}

Received: 1 September 2020; Accepted: 9 October 2020; Published: 19 October 2020

check for updates

\begin{abstract}
With the introduction of the more electric aircraft, there is growing emphasis on improving overall efficiency and thus gravimetric and volumetric power density, as well as smart functionalities and safety of an aircraft. In future on-board power distribution networks, so-called high voltage DC (HVDC, typically +/-270VDC) supplies will be introduced to facilitate distribution and reduce the associated mass and volume, including harness. Future aircraft power distribution systems will also very likely include energy storage devices (probably, batteries) for emergency back up and engine starting. Correspondingly, novel DC-DC conversion solutions are required, which can interface the traditional low voltage $(28 \mathrm{~V}) \mathrm{DC}$ bus with the new $270 \mathrm{~V}$ one. Such solutions presently need to cater for a significant degree of flexibility in their power ratings, power transfer capability and number of inputs/outputs. Specifically, multi-port power-scalable bi-directional converters are required. This paper presents the design and testing of such a solution, addressing the use of leading edge wide-band-gap (WBG) solid state technology, especially silicon carbide (SiC), for use as high-frequency switches within the bi-directional converter on the high-voltage side.
\end{abstract}

Keywords: DC-DC converters; multi-port dual-active bridge (DAB) converter; wide-band-gap (WBG) semiconductors; silicon carbide ( $\mathrm{SiC}$ ) MOSFETs; power converter

\section{Introduction}

The use of $115 \mathrm{~V}_{\mathrm{AC}} 400 \mathrm{~Hz}$ and $28 \mathrm{~V}_{\mathrm{DC}}$ power networks is a historical feature of avionic electrical power generation and distribution systems. The AC power is used directly for high power loads, such as starting, and is then rectified and conditioned to supply the bus power of $+28 \mathrm{~V}_{\mathrm{DC}}$ distributed to aircraft control systems, flight decks, and entertainment systems. However, there has been a pronounced movement within the aerospace industry to shift towards cleaner, more efficient and lower maintenance aircraft design as a result of the emergence of high fuel prices, the global warming problem, and high operating costs. The electrification of the aircraft to replace hydraulic or pneumatic functions with electrical ones is one of the prime movers in this field, as in the concepts of the More and All electric aircrafts [1-3]. This refers in particular to the replacement by electric actuators of complex aircraft hydraulic actuator systems, thereby dramatically reducing weight, maintenance costs, fuel consumption, footprint of carbon dioxide, and operating costs. Furthermore, the removal of pneumatic engine bleed systems makes it possible to run the engine more effectively and thus to save additional fuel.

The replacement of hydraulic and pneumatic systems with electric actuators and systems greatly increases the total electrical power requirements for an aircraft, requiring new approaches to the safe and intelligent delivery of aircraft power. To achieve the higher power ratings in a feasible way, 
novel enhanced aircraft Electrical Power Distribution System (EPDS) architectures investigated over recent years include smart power management systems, characterized by the presence of at least one high-voltage dc bus (HVDC, +/-270 V) and a low-voltage DC bus (LVDC, typically, $28 \mathrm{~V}$ ), as illustrated very summarily, for instance, in Figure 1 . Such architectures require the presence of HVDC/LVDC bi-directional DC-DC converters to interconnect and manage power transfer between the two bus levels, also enabling the addition of energy storage devices. Due to the abundance and variety of loads and still partly undefined power ratings (e.g., batteries) here, the focus is on a solution which enables a high degree of flexibility in relation to power scaling. In particular, a solution is pursued, in which a basic DC-DC converter power cell can be paralleled a number of times with control and supervision functionalities carried out by a unique central board. The prime drivers of the design are of course efficiency and power density, with an eye also to solutions meeting the single-fault tolerance expectations typical of avionic solutions. The design and test results of both single power cell and parallel operation are presented.

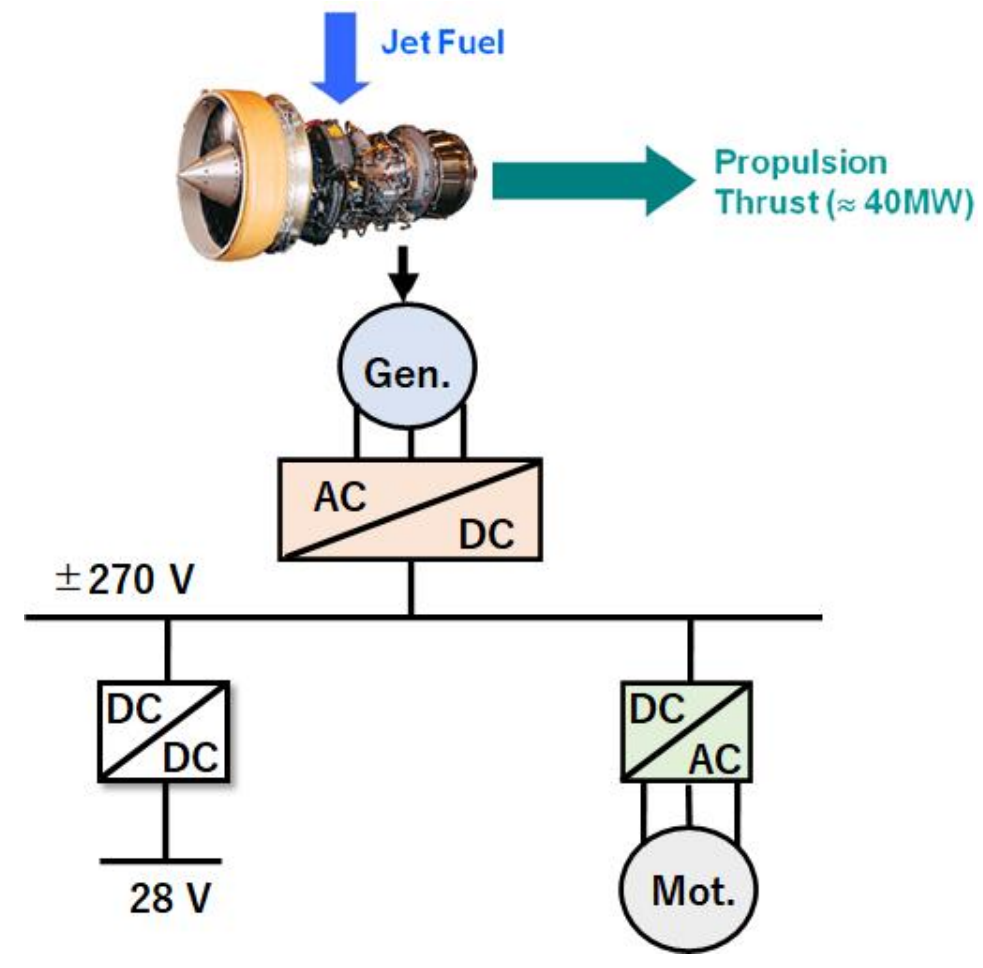

Figure 1. Generic illustration of future aircraft electrical network, including an high voltage dc (HVDC) power distribution bus: dedicated bi-directional DC-DC converters interface the 270 and $28 \mathrm{~V}$ buses; inverters cater for the AC loads (e.g., motors in pumps, actuators). Additional ports in the DC-DC converters may also be used to interconnect storage devices, with different voltage levels.

\section{270-28 V Bi-Directional Converter Design}

The dual-active-bridge (DAB) topology was chosen among different solutions, for the following main advantages in this context $[4,5]$ :

- 1 st order dynamics and current generator equivalent characteristics, which enable straightforward parallel-ability and thus: (a) overall power scalability; (b) in-built redundancy and guarantee of reduced power operation capability; (c) overall efficiency optimization taking into account maximum efficiency versus load values of individual converter cells;

- easy extension to multi-port realization to interconnect, for instance, to storage devices [6];

- Zero-Voltage-Switching (ZVS) turn on of the power devices over a broad load range;

- suitability for use of planar magnetics design with possibility of integrated magnetics solutions; 
- intrinsic current limitation in case of output short-circuit fault without the need for additional limiters or breakers.

DAB converter theory and operation is amply covered in literature already (see [2,3,6-8], for example) and so, here, the treatment of fundamental aspects is limited to some essential points of relevance to the subsequent discussion. For simplicity, we refer to the $270 \mathrm{~V}$ bus side as the input and the $28 \mathrm{~V}$ side as the output, but the discussion holds equal if the two are interchanged. The DAB consists of two full H-Bridges interconnected through a transformer, Figure 2a. The switches in diagonal pairs of either H-bridge are always turned on and off jointly and, within each bridge, each diagonal pair has $50 \%$ duty ratio. Control of output current delivery is achieved by introducing a phase-shift between the operation of the switches in the two H-bridges; with a series inductance all referred to the primary side, as in Figure 2a, the transformer secondary side gets directly connected to the output capacitor and so, the output voltage value is imposed back onto the primary scaled by the transformation ratio; the algebraic sum of input voltage and primary-reflected output voltage determines the voltage falling across the series inductor $L_{C}$ during the phase-shift interval. Therefore, $L_{C}$ can be effectively used as the current control element and its stored energy helps achieve turn-on soft switching of the power devices by means of resonance phenomena with the parasitic capacitance of the switches during the transitions. Both the power value and flow direction can be controlled by intervening on the value of the phase-shift $\Phi$ between the driving signals of the input and output H-bridges.

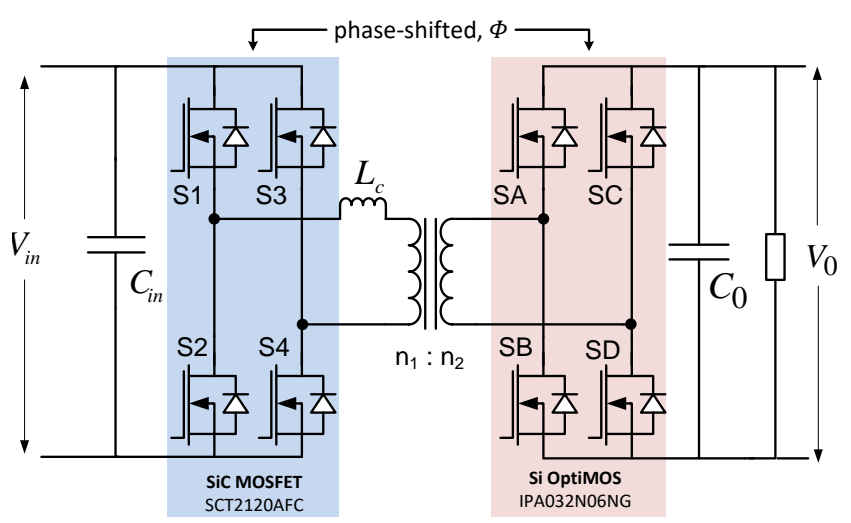

(a)

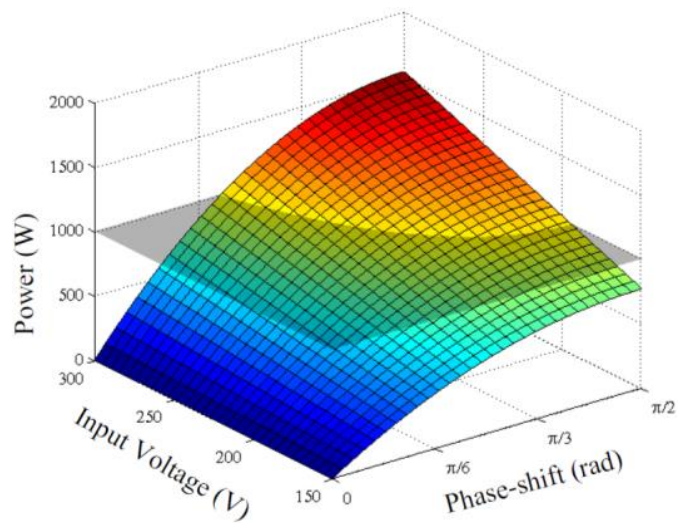

(b)

Figure 2. Main features of Dual-Active-Bridge converter: circuit schematic of the converter topology based on SiC MOSFETs or the primary and Si MOSFETs for the secondary side (a) and summary of output power delivery as a function of input voltage and phase-shift (b).

According to the fundamental model and neglecting losses, the power flow can be expressed by:

$$
P_{D C}=\frac{V_{I N} \cdot V_{O}}{n \omega_{s} L_{C}} \cdot \Phi\left(1-\frac{\Phi}{\pi}\right)
$$

where $V_{I N}, V_{O}$ are the values of the input and output DC voltages, $n$ is the transformer turns ratio, $\omega_{s}=2 \pi f_{s}\left(f_{s}\right.$ switching frequency), and $\Phi$ is the phase-shift (in radians). Equation (1) corresponds to a parabolic power delivery as a function of the phase-shift, with maximum power capability influenced by the value of input voltage, as summarized in Figure $2 \mathrm{~b}$ considering a realistic variable input voltage range for the intended application.

\subsection{Power Cell Design}

Here, the intended nominal power rating of the brick power cell is $1.2 \mathrm{~kW}$ : more bricks can be connected in parallel to scale the power up. The primary $(270 \mathrm{~V})$ and secondary $(28 \mathrm{~V})$ side power cells are implemented using $650 \mathrm{~V}$ SiC MOSFETs (SCT2120AFC, ROHM Ltd., Kyoto, Japan) and $60 \mathrm{~V}$ 
Opti-MOS (IPA032N06N3 G, Infineon Technologies, Munich, Germany), respectively. The choice of $\mathrm{SiC}$ devices for the primary (i.e., high voltage) side, as opposed to $\mathrm{Si}$ ones, is mainly motivated by the following reasons and advantages:

- Possibility to use a MOSFET type transistor as opposed to an IGBT, which allows for faster switching, removes the need for anti-parallel free-wheeling diode connection, and enables for more symmetrical operation in the forward and reverse power flow directions;

- Higher switching frequency capability with contained impact on efficiency, enabling the achievement of enhanced power density figures;

- Better temperature stability and higher temperature capability, which yield both more temperature independent efficiency levels over the intended operational range and favor long term reliability;

- Smaller overall intrinsic capacitances, which allow to achieve tun-on ZVS operation down to lower load values.

The gate drivers are bespoke designed, using non-symmetrical drive input voltage voltages for the $\mathrm{SiC}$ transistors on the primary side of -4 to $+20 \mathrm{~V}$ and -4 to $+15 \mathrm{~V}$ for the low voltage Si MOSFETs on the secondary side. Whereas a non-symmetrical drive voltage is a strict functional requirement of $\mathrm{SiC}$ MOSFETs, the choice to also apply an unsymmetrical driving voltage for the secondary-side $\mathrm{Si}$ transistors merely responds to cost containment targets, by enabling the design and production of a single gate-drive circuit, which can be adapted for both transistor types simply by replacing two ICs and one resistor in the circuit, with identical footprint. It should be noted moreover, that applying a somewhat higher (i.e., closer to zero) off-state bias voltage to the Si MOSFETs also allows to achieve faster switching transitions, with slightly reduced losses. Whereas the impact on overall efficiency is contained, the electro-thermal stress reduction in the transistor is interesting. For reference, the gate driver circuit schematic is shown in Figure 3. Insulation at gate-driver level is achieved by means of opto-couplers and signal transmission between control board and power cell is by means of optical fibers, ideal for avoiding electro-magnetic interference issues in reliability critical applications.

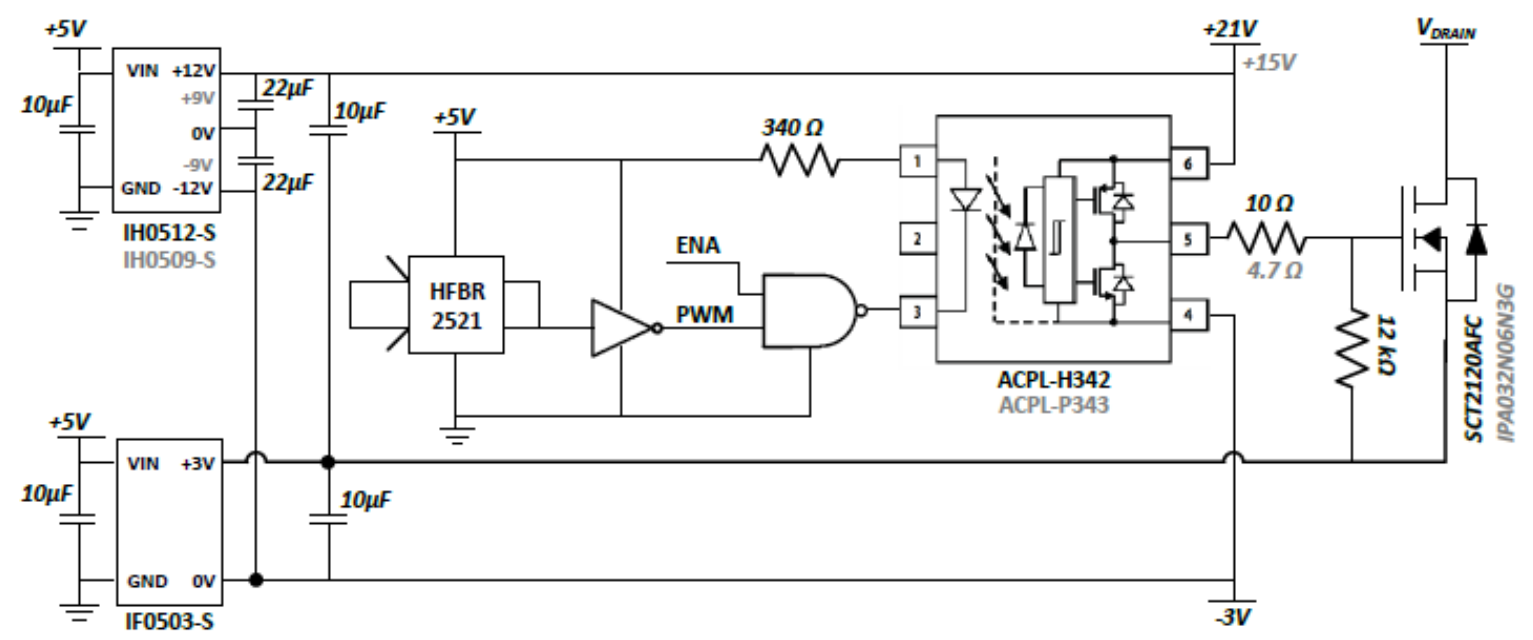

Figure 3. Schematic of gate driver circuit, featuring a unified design for both primary and secondary side transistors.

The magnetics are the other core components of the converter: the transformer provides galvanic isolation and voltage matching, the voltage across the series of its leakage inductance and the external inductor determines the current waveform flowing through the circuit. Since the transformer turns-ratio is relatively low, planar design of transformer and inductor are feasible and greatly interesting for increasing power density and improve thermal management; the components, shown in Figure 4, were custom designed and manufactured externally based on in-house specification. It is worth 
noting that the inductor could be realized as leakage inductance of the transformer, in the form of integrated magnetics. However, since its value is a key parameter of the converter control and dynamic characteristics, as well as of the ZVS load range, keeping the two components separate enables easier design optimization and better transformer thermal management. The switching frequency was chosen to be $100 \mathrm{kHz}$ to start with and the transformer turns ratio was 19:2. After fixing the switching frequency and the turn ratio values, the maximum power and ZVS load range depend on the series inductor and the controllable phase-shift. Hence, an inductance value capable to satisfy the power constraints needs to been selected.

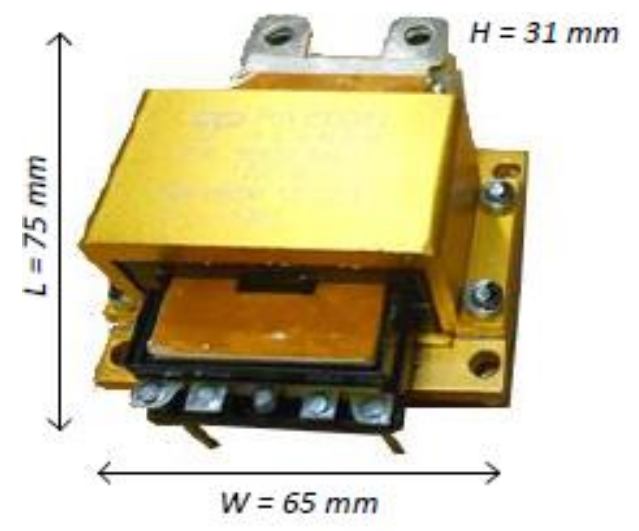

(a)

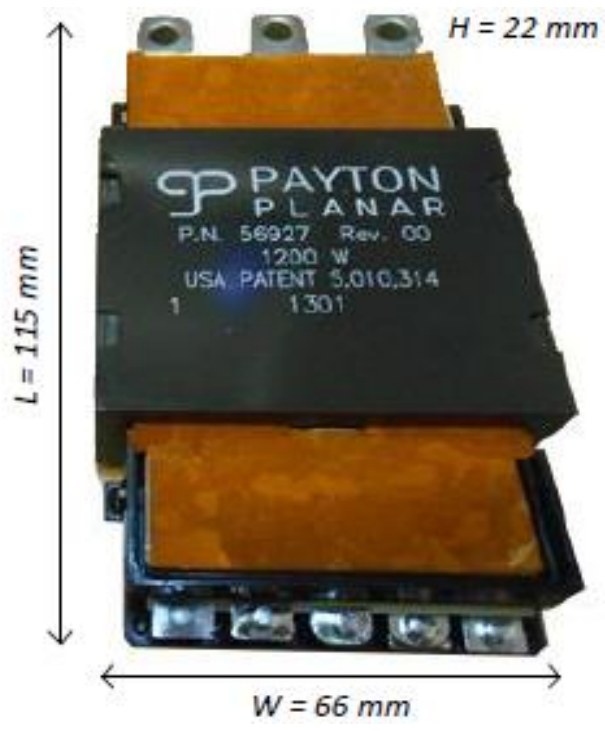

(b)

Figure 4. Photograph of planar magnetic components: heat-sinked inductor, (a), and transformer, (b).

\subsection{Control Design}

The goal of the control strategy is to keep the DC bus voltage constant at the desired voltage reference $(28 \mathrm{~V})$ in both forward and backward power flow, adjusting the phase-shift between the primary and secondary bridge. Starting from the fundamental reduced model of the DAB converter shown in Figure 5 along with the representative voltage and current waveforms illustrating basic operation and control of the converter, a state-space-average (SSA) model of the system was developed.

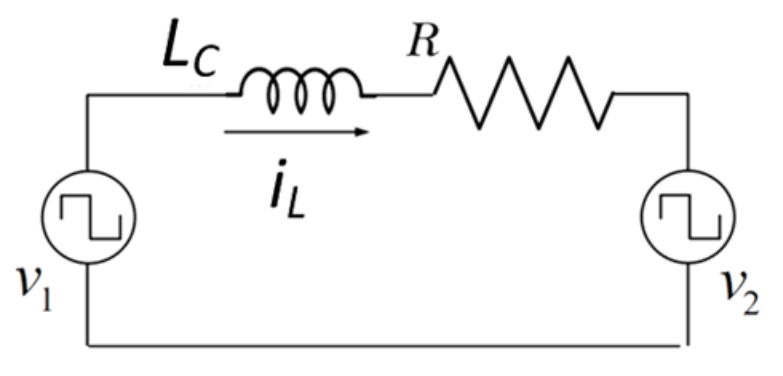

(a)

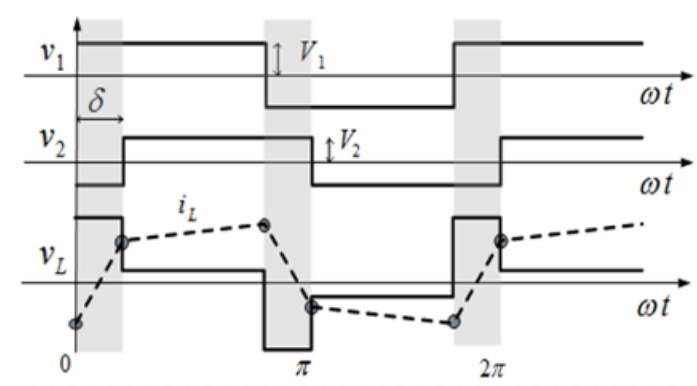

(b)

Figure 5. Dual-active bridge (DAB) basic equivalent state-space average model (a), and characteristic voltage and current waveforms illustrating the control principle (b).

The open loop dynamic characteristics of the converter are shown in Figure 6, in terms of its Bode-plot gain and frequency Bode-plots. It is important to underline that the converter DC gain, 
bandwidth and phase-margin are load-dependent, an aspect which needs to be duly taken into account when closing the loop.

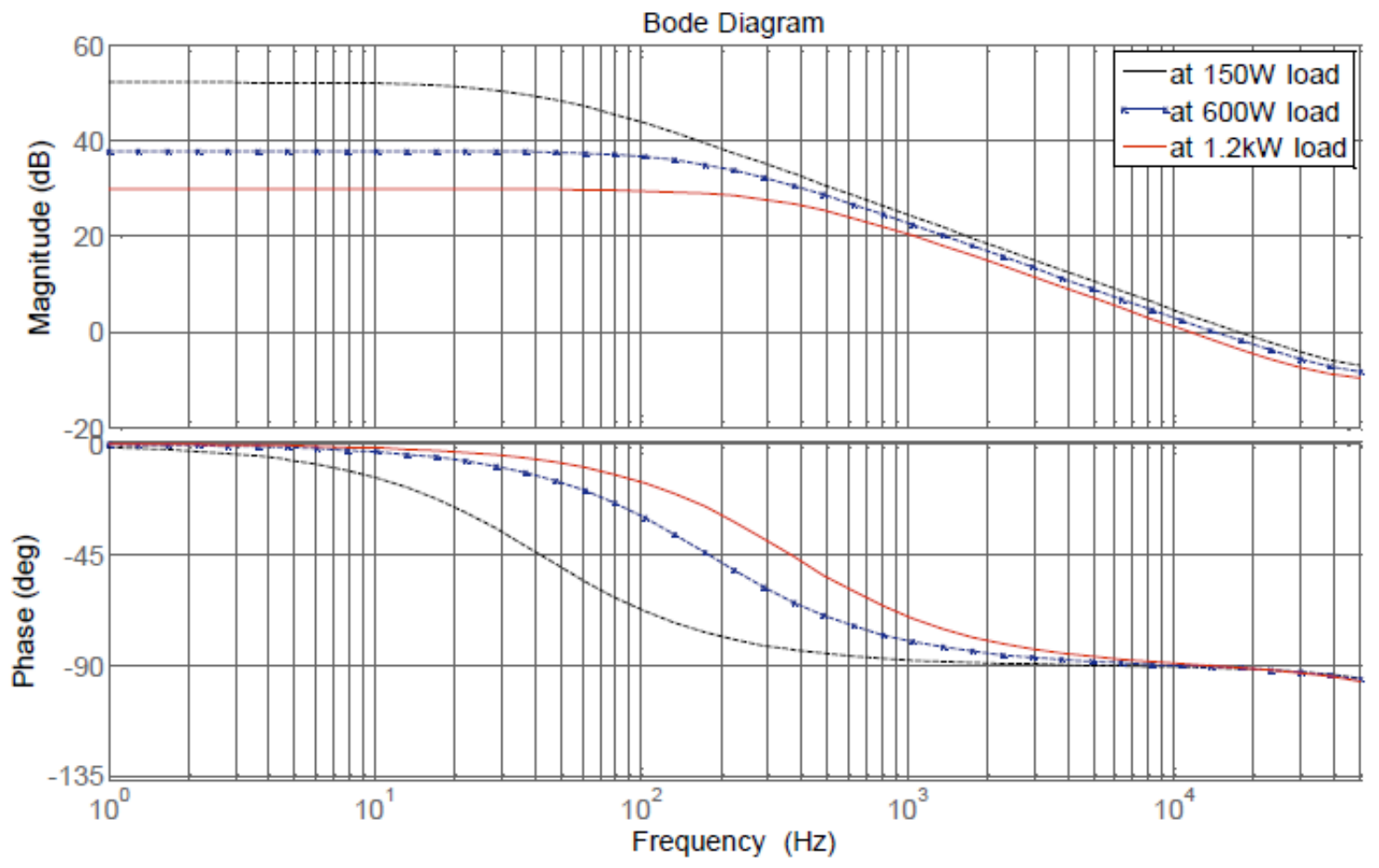

Figure 6. Open-loop Bode-plots of gain and phase for the power converter cell.

The chosen control system block diagram is shown in Figure 7. It consists of DC bus voltage control loop with a feed-forward current loop. The measured voltage $V_{O}$ is compared with the reference voltage $(28 \mathrm{~V})$ and the error is fed through a PI controller to generate the desired phase-shift between the primary and secondary voltage square-waves. A feed-forward phase-shift compensation is added to make the system response faster. Based on the analytical model of the converter, the PI parameters are chosen to obtain the desired bandwidth with a proper phase margin. The phase-shift $\Phi$ depends non-linearly on load resistance $R_{O}$ as:

$$
\Phi=\frac{1-\sqrt{1-\frac{8 n f L_{c} V_{o}}{V_{i} R_{o}}}}{2}=\frac{1-\sqrt{1-\frac{8 n f L_{c} I_{o}}{V_{i}}}}{2}
$$

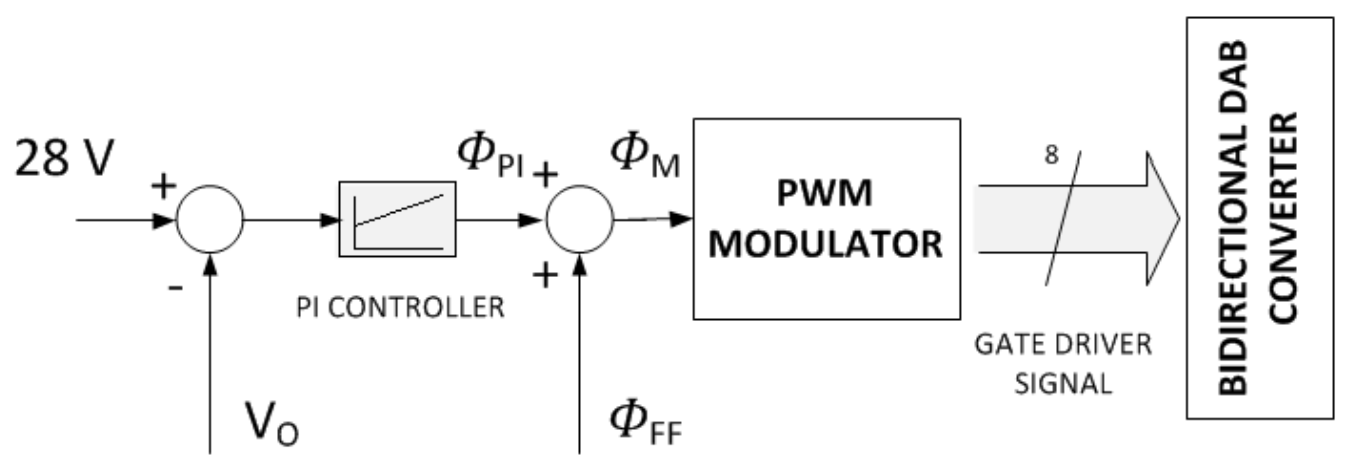

Figure 7. Converter control scheme with both a feedback and a feed-forward loop. 
Since the switching frequency and the inductance are known, the output current and the input voltage are acquired by the sensors, it is possible to estimate the proper phase-shift during the working operations and let the PI controller manage only the small variations. Simulation results for the closed-loop response with the introduced control are summarized in Figure 8a, while in Figure $8 b$ the converter step response for different start-up power levels is reported. It is clear that load dependence of the dynamics characteristics is significantly contained with the chosen approach.

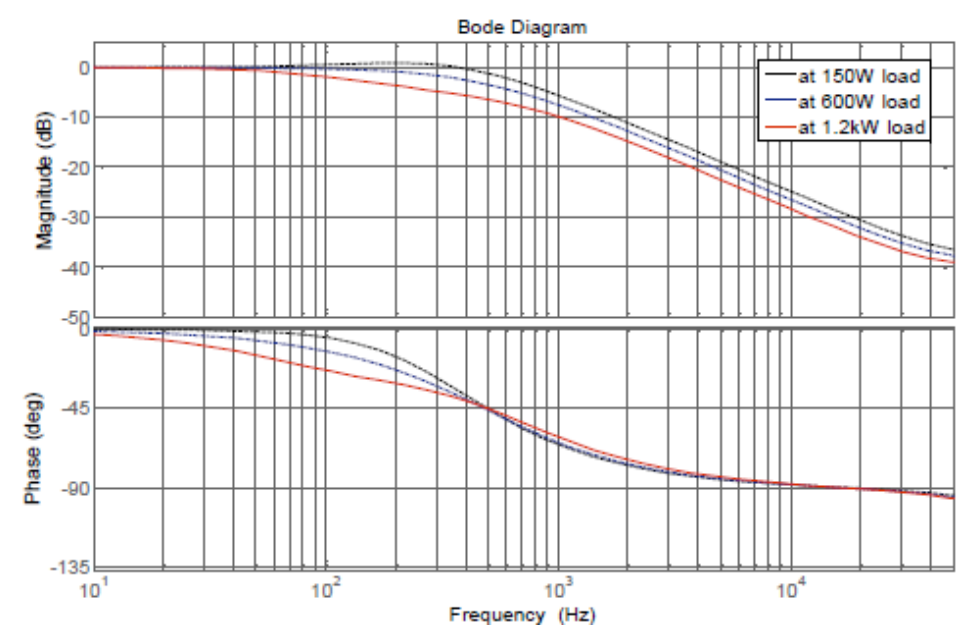

(a)

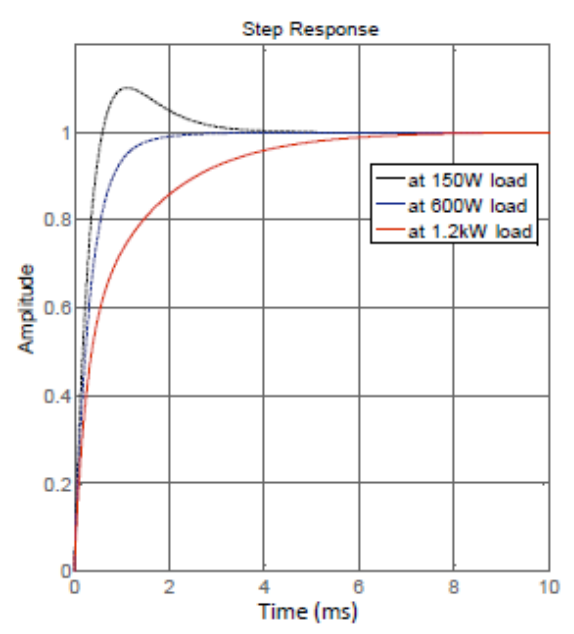

(b)

Figure 8. Dynamic response characterization of the converter (simulation results): closed-loop Bode plots (a) and system step response at different power levels (b).

The design phase also addressed EMI filtering on the $270 \mathrm{~V}$ side, ensuring compliance of standard avionic requirements (e.g., MIL-STD-704F) with a standard and compact damped LC filter design.

\subsection{Experimental Characterisation}

Figure 9 shows the engineered TRL6 level DC-DC converter, including control platform and flight-like enclosure, with details of connectors and wiring. Figure 10a shows some representative voltage and current waveforms at about $50 \%$ load $(600 \mathrm{~W})$ and Figure $10 \mathrm{~b}$ summarizes measured efficiency data, still at $600 \mathrm{~W}$, as a function of the input voltage in the range 230-290 V. Efficiency over load in the forward and backward power flow direction is reported in Figure 11: as can be seen, in the design and engineering of the converter, attention was taken to ensure high performance at relatively low loads; such approach is dictated by the actual mission profile of the converter, which foresees low-load operation as the most frequent condition, with the requirement to deliver the full power capability limited only to very short time durations. Therefore, it is very important to consider energy (as opposed to power) efficiency; that is, the integral over time of the power losses at the various load conditions to maximize overall aircraft efficiency. Moreover, it should be noted that due to the intended parallel operation of the converters, more units can be made to work at an optimized efficiency level even when the full capability of one single converter is not exhausted: for instance, if the total power demand is $900 \mathrm{~W}$, it is more efficient to run tow cells in parallel than a single cell. The above represent a novel approach to design efficient power conversion systems, which will become increasingly important as the electrification level increases. It is also worth noting here, that bi-directional operation is mainly requested for enabling the possibility to use batteries connected on the lower-voltage side to be used as power source during some abnormal operational regimes or to implement regenerative energy storage functionalities. Therefore, the forward power transfer direction is still to be regarded as the primary one for the characterization for the converter. 


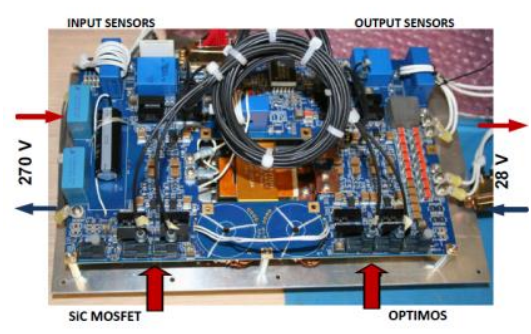

(a)

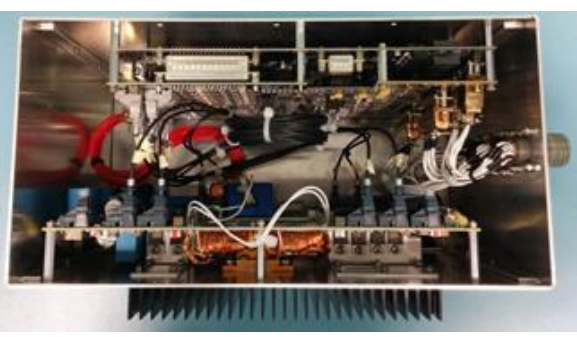

(b)

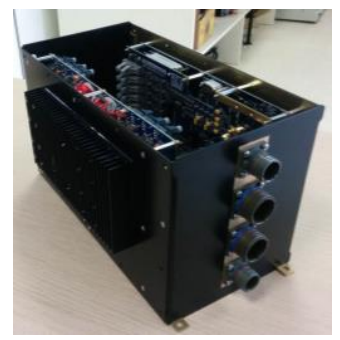

(c)

Figure 9. Photographs of developed hardware: single DC-DC converter cell (a); view of cased converter and control board, with details of wiring, and connectors (b); external view of cased unit (c).
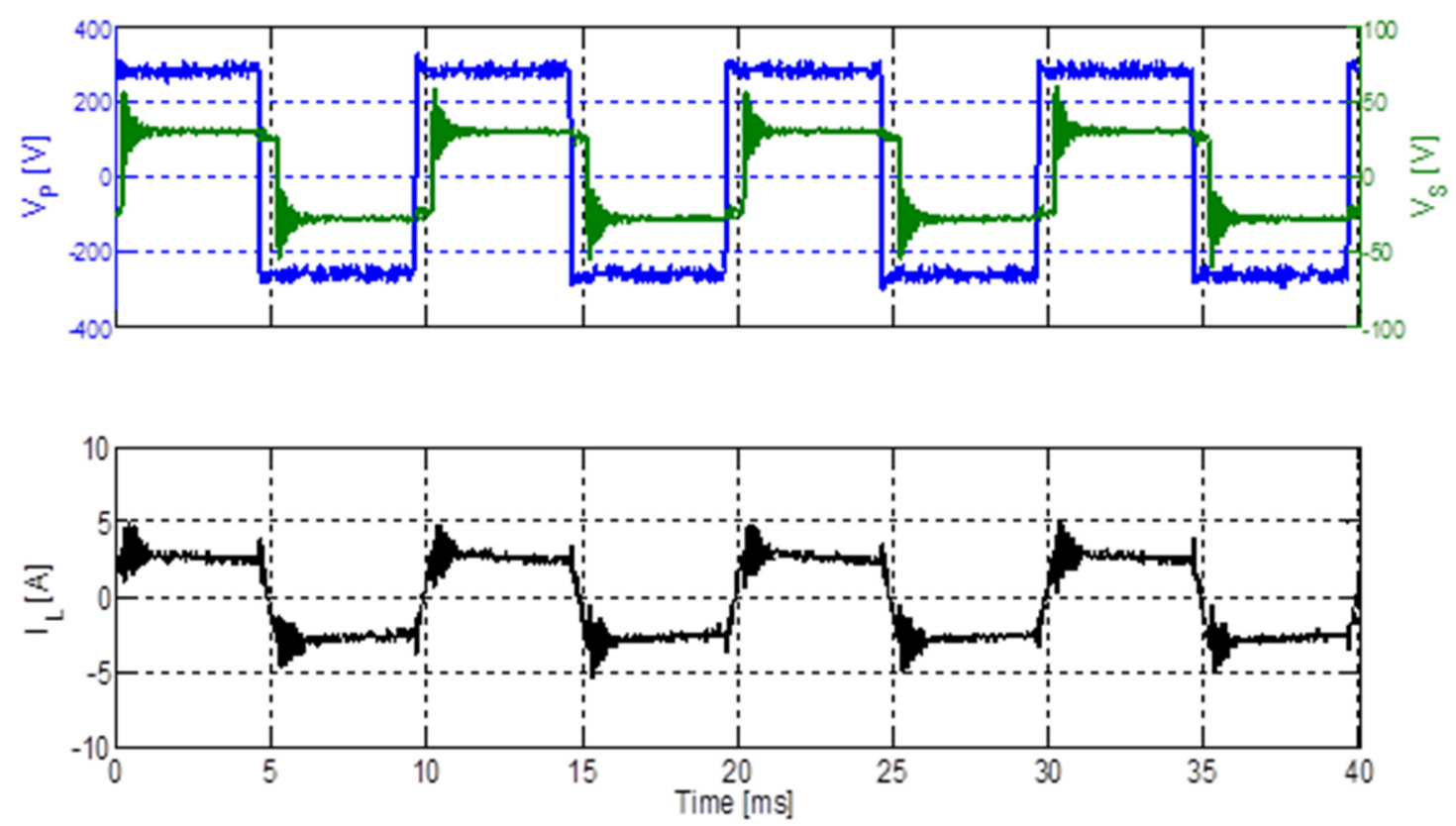

(a)

\begin{tabular}{|ccc|ccc|c|c|}
\hline \multicolumn{7}{|c|}{ Efficiency measurement for variable input voltage } \\
\hline $\mathbf{V}_{\text {IN }}$ (V) & $\mathbf{I}_{\text {IN }}$ (A) & $\mathbf{P}_{\text {IN }}$ (W) & $\mathbf{V}_{\text {OUT }}$ (V) & $\mathrm{I}_{\text {OUT }}$ (A) & $\mathrm{P}_{\text {out }}(\mathrm{W})$ & $\mathrm{P}_{\mathrm{D}}(\mathrm{W})$ & $\boldsymbol{\eta}$ \\
\hline 235 & 2.8 & 653.0 & 28.1 & 21.2 & 597.1 & 55.86 & $\mathbf{9 1 . 4}$ \\
240 & 2.7 & 650.0 & 28.1 & 21.3 & 597.8 & 52.20 & $\mathbf{9 2 . 0}$ \\
250 & 2.6 & 657.5 & 28.2 & 21.6 & 608.6 & 48.91 & $\mathbf{9 2 . 6}$ \\
260 & 2.5 & 650.8 & 28.2 & 21.5 & 607.3 & 43.48 & $\mathbf{9 3 . 3}$ \\
270 & 2.4 & 645.6 & 28.3 & 21.5 & 606.5 & 39.01 & 94.0 \\
280 & 2.3 & 641.3 & 28.3 & 21.4 & 605.6 & 35.78 & 94.4 \\
290 & 2.2 & 638.2 & 28.3 & 21.4 & 604.5 & 33.74 & 94.7 \\
\hline
\end{tabular}

(b)

Figure 10. In (a), transformer primary and secondary voltage waveforms (top) and series inductor current (bottom); in (b), efficiency and detail of average voltage and current values. 


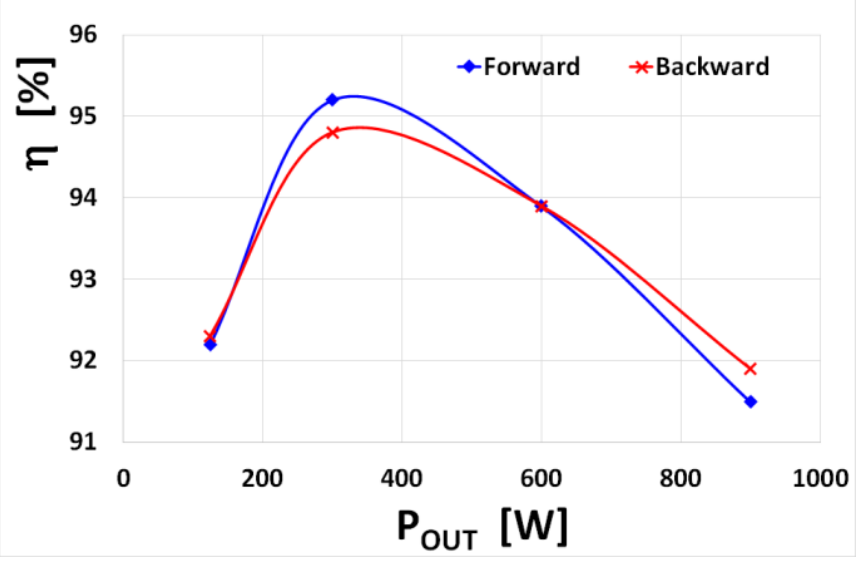

Figure 11. Measured converter efficiency in the forward and backward power transfer mode.

It should be noted that, although some ringing due to parasitic inductance is visible on the secondary side voltage waveform, no snubbers were eventually used in the converter implementation due to the Si MOSFETs being fully avalanche rated by design and not giving signs of any degradation over extended test periods for the parasitic energies involved in the switching transitions here. Indeed, the transistors are never driven into avalanche up to full load.

Some examples of closed-loop dynamic response are reported in Figure 12. Tests on the prototype confirmed the anticipated benefits of the chosen control approach, with consistent performance for both directions of power flow: the presence of the additional feed-forward loop is profitable and enables the converter to promptly manage large and rapid changes of power demand without unacceptable rising or falling of the voltage on the $28 \mathrm{~V}$ bus.
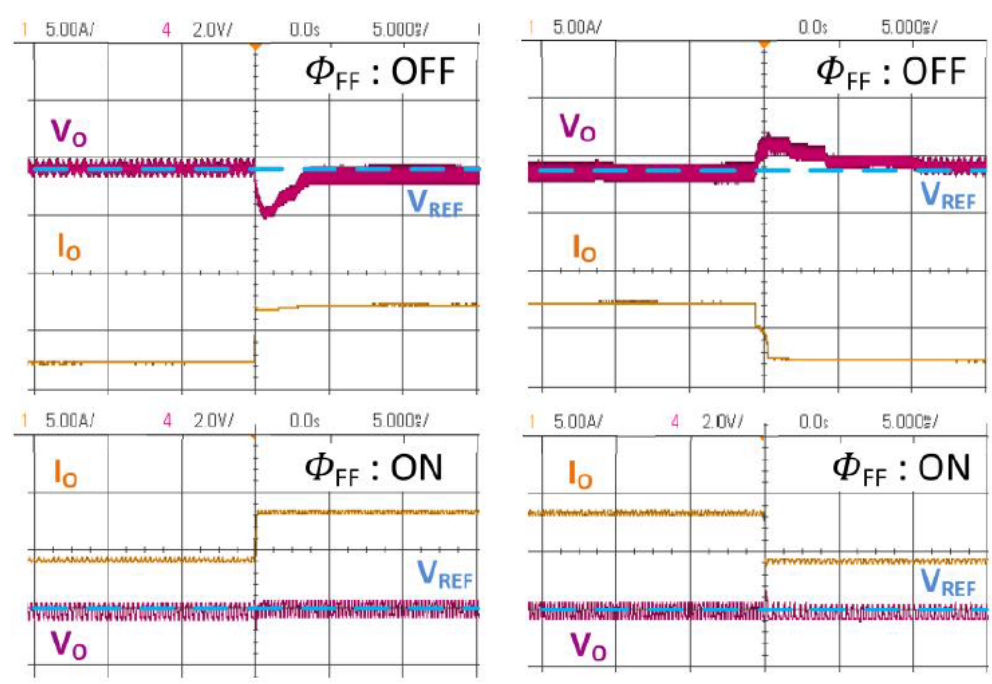

(a)

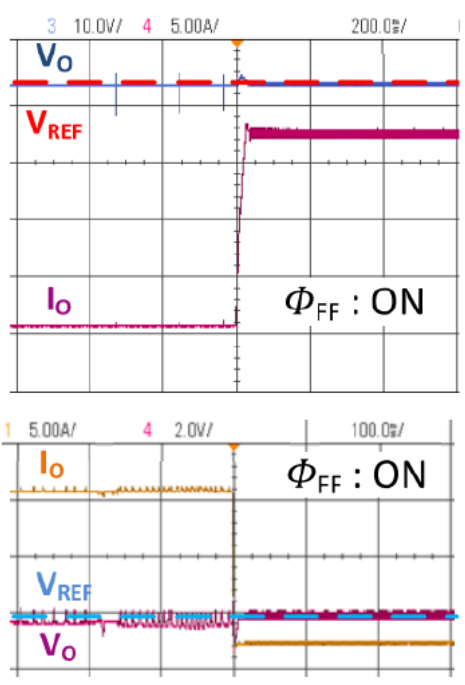

(b)

Figure 12. Scope snapshots of output voltage variation in the presence of abrupt and large step changes in the load ( $\pm 5 \mathrm{~A}$ in (a) and $\pm 15 \mathrm{~A}$ in (b)), without (top) and with (bottom) feed-forward loop, respectively.

\section{Converter Paralleling for Power Scalability}

To ensure a modular system architecture, with all its associated benefits, the parallel operation of more converters was further considered. The basic straightforward parallel connection of two converter units directly linked to the load is illustrated with the help of Figure 13. Due to its current source 
dynamic characteristics, paralleling of DAB converters is relatively straightforward and bi-directional converter (BDC) units are theoretically capable of working together without any additional control [9]. However, as soon as an unbalance arises, there is no way to equally split the power among the two modules without control. That is clearly shown in the results of Figure 14a,b. Moreover, a potential drawback is related to the bi-directional nature of the DAB converter: an issue could arise if the balance is established with one converter that works in regenerative configuration, as per experimental results of Figure 14c,d. In such a worst-case scenario, the paralleling of two units is not only pointless but even potentially destructive. For those reasons, a technique is needed for proper interconnections of BDC units.

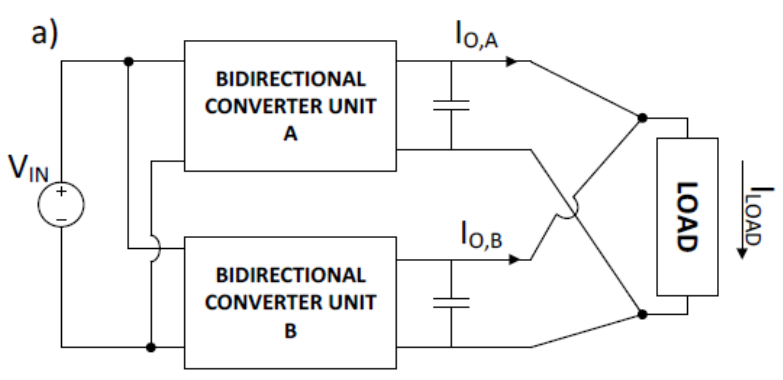

(a)

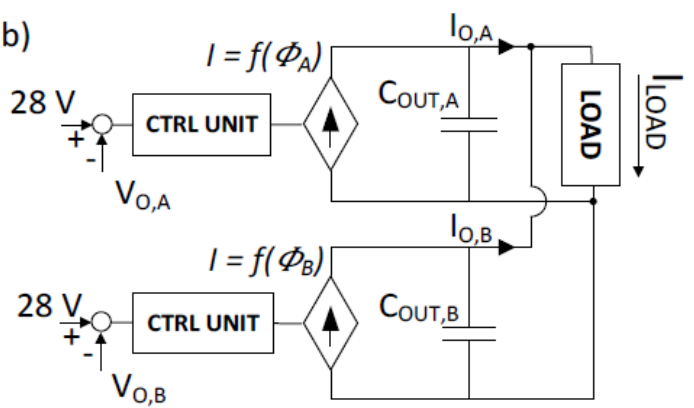

(b)

Figure 13. (a) Parallel connection of 2 BDCs; (b) controlled current source equivalent model.

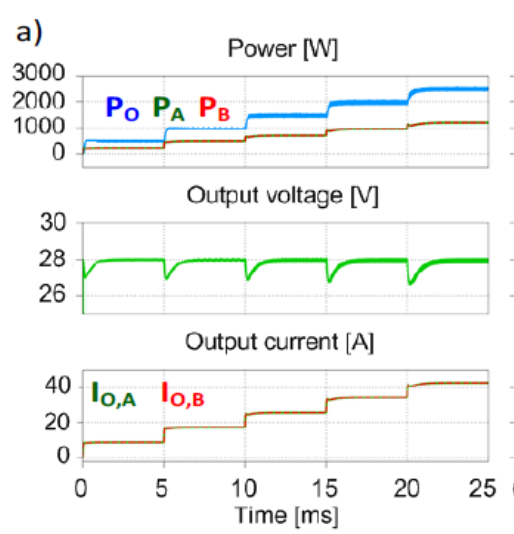

b)

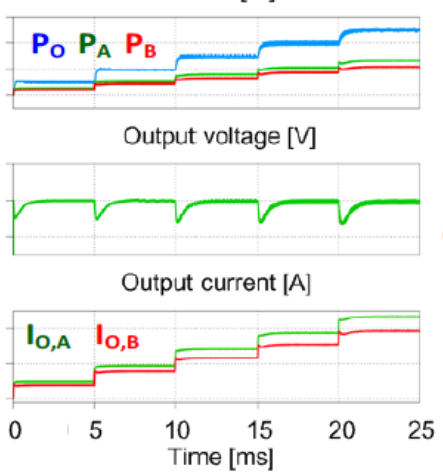

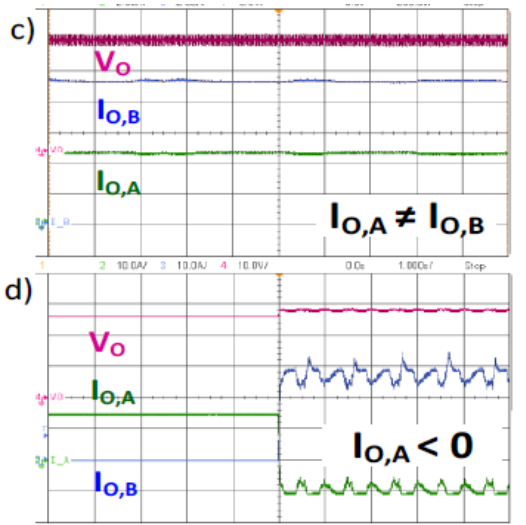

Figure 14. Parallel connection of BDC units without additional control: balanced, (a), and unbalanced, (b) conditions in simulation; balanced (c) and unbalanced (d) conditions in experimental tests.

Therefore, in this configuration, the aim of the control was still to regulate the load voltage, but also to ensure proper sharing of power delivery between the two modules. The corresponding equivalent control scheme in this case is illustrated in Figure 15. The methodology employed here consists in decoupling the control loops: one converter, acting as the master unit, ensures control of the output voltage to a constant value with the modality described in the previous section; the other, acting as a slave unit, is in charge of power sharing and operates in current control mode. The output current of each converter is sensed and the information is shared via a dedicated communication bus. The voltage controller in the salve unit is disabled and the master output current is fed-back as the set-point of the slave feedback-loop to determine the phase-shift. The feed-forward loop can also be added with a similar approach for both modules. 


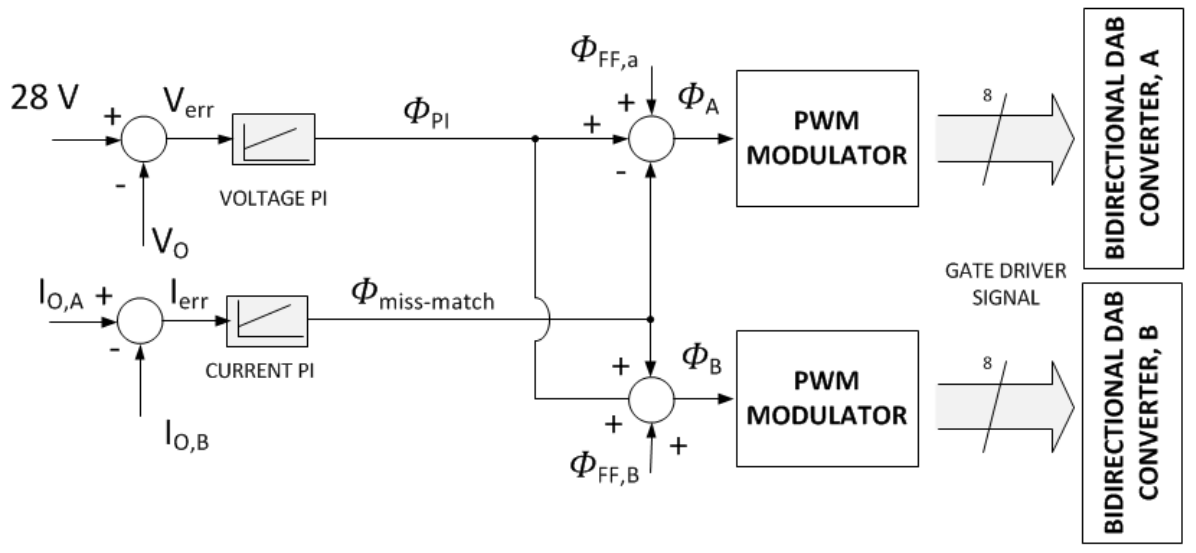

Figure 15. Structure of closed-loop system for parallel operation with current compensator.

Figure 16 shows the results obtained on the lab-prototypes for different power requests, after the implementation of this method. The tests confirm that the management of the energy is realized in good agreement with simulations and the output voltage is well regulated. The main advantage of the proposed solution is the fully controllable load sharing, which can be achieved with great accuracy in a simple and straightforward manner; the main limitation to its deployment is the need to exchange information between converters, which can become problematic in the case of multiple parallel modules if fast dynamic response is required. Loss of the communication-link or failure of the master unit would result in system shut-down.
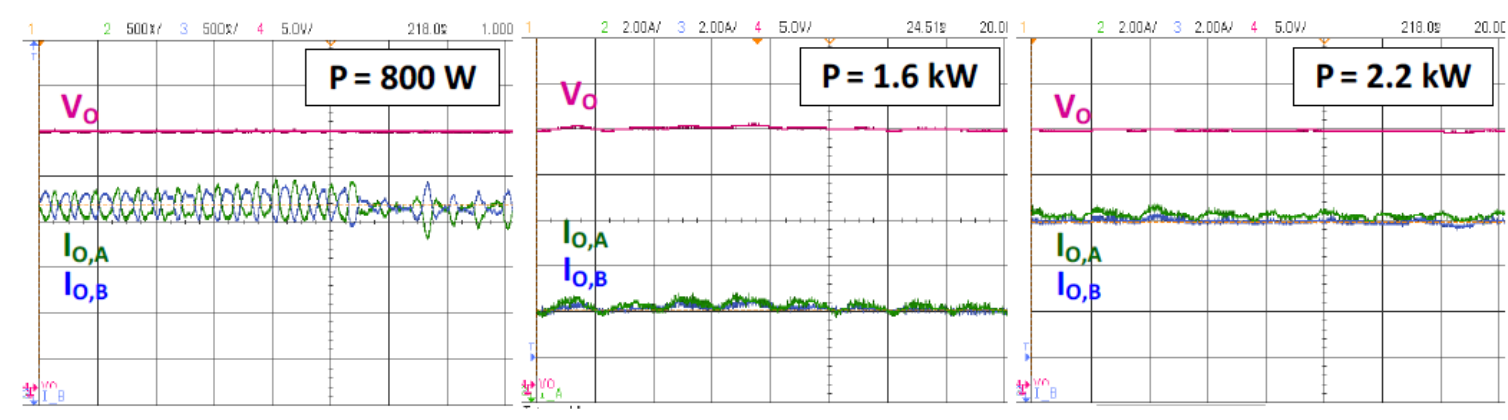

Figure 16. Experimental results under varying loads when using the current sharing parallel method.

Droop control is also a well-known practice used in power systems to share power among different generators. This concept has been recently proposed for other kinds of applications, such as speed control for integrated modular motor drives or modular DC/DC converter for smart transformers $[10,11]$. In this case, the droop control, illustrated in Figure 15, could be a suitable solution to enable parallel connection and to allow power sharing among the DABs; moreover, it does not require any communications between the BDC modules. The concept is to add a virtual resistor $\left(K_{d r o o p}\right)$ into the feedback loop, which drops the internal voltage set-point $\left(V_{O, r e f}\right)$ as a linear function of the output current $\left(I_{0}\right)$

$$
V_{o, r e f}=V_{o, r e f}^{*}-K_{d r o o p} \cdot I_{o}
$$

Through this strategy, each converter exhibits a self-balancing characteristic, so it is not necessary for the single unit to be aware of the other elements in the system. The undesired effect of a steady-state error on the regulated voltage could be reduced or completely removed by another PI regulator, as also depicted in Figure 17. 


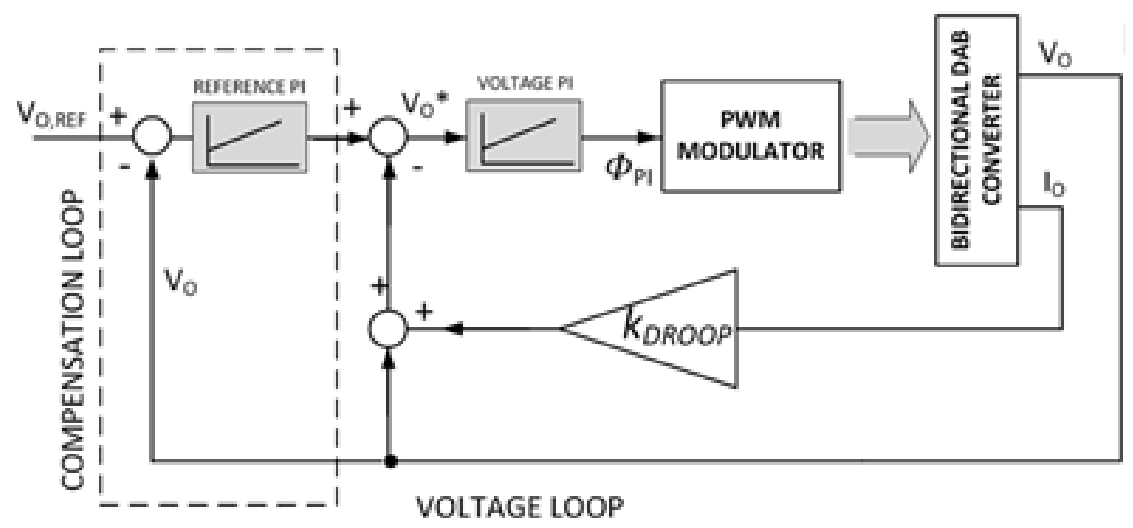

Figure 17. Block diagram illustration of the voltage-droop control with reference compensation protocol.

The results in Figure 18 show each converter working independently with the proposed voltage droop method, which intrinsically equalizes the power flow between the two BDC units connected in parallel to a resistive load. As the ratio of the droop coefficients is directly related to the amount of current flowing in each module, an asymmetrical power distribution is also possible simply adopting different virtual resistors for the single units (Figure 18b,c). In the system under analysis, the outer loop cut-off frequency was around one-third that of the inner loop.

a)

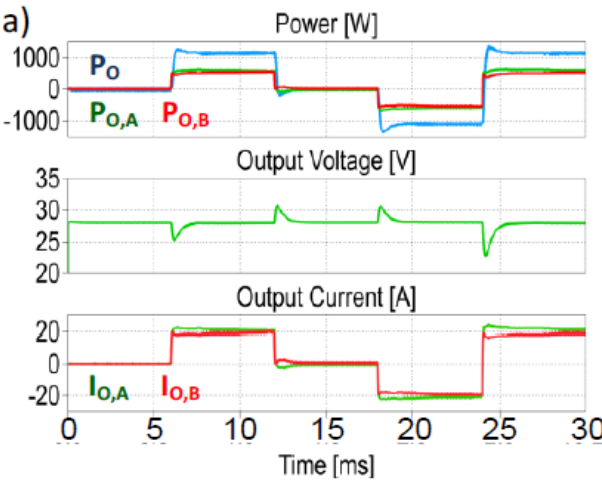

b)

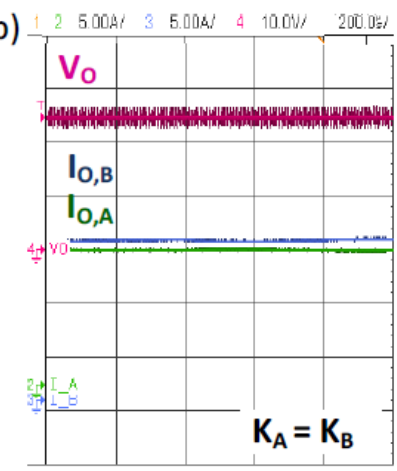

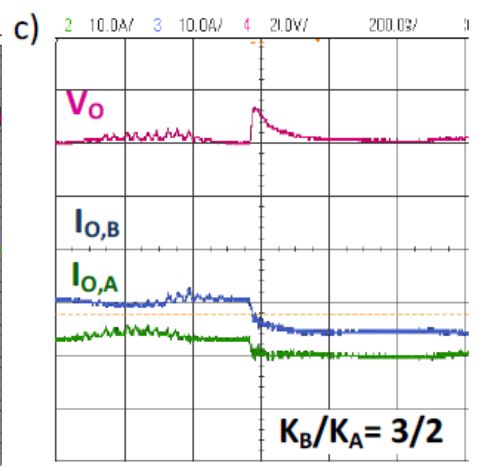

Figure 18. Parallel operation with droop control: converters working independently (a) and details of current unbalance between the two in the steady-state $(\mathbf{b})$ and transient (c) regimes.

\section{Conclusions}

This paper has presented the design and development of a solution for implementing power-scalable bi-directional DC-DC conversion in future aircraft power networks [12,13]. The solution is based on the use of a dual-active bridge topology, which can easily be extended to multiple ports versions when the need to interface additional elements, such as batteries for storage, emerges [6,14]. In addition, the topology is suitable for the use of novel semiconductor technologies on the high-voltage side; in particular, silicon carbide ( $\mathrm{SiC}$ ) MOSFETs can yield important gains in switching frequency capability and converter performance optimization in terms of efficiency and thermal management requirements $[14,15]$. In the future, the possibility to develop bespoke modules will enhance the potential for disruptive progress in the integration level that can be realistically achieved [16].

Author Contributions: A.L. co-funded and managed the R\&D activity and ensured technical lead in the engineering phase to TRL6 level; F.G. was the person in charge of the design and testing of the converter prototype and co-authored the paper; A.C. co-funded and supervised the research and development activity and co-authored the paper. All authors have read and agreed to the published version of the manuscript.

Funding: This research and development activity was partly funded by the EU FP7-JTI via the REGENESYS project (ID 308129).

Conflicts of Interest: The authors declare no conflict of interest. 


\section{References}

1. Buticchi, G.; Bozhko, S.; Liserre, M.; Wheeler, P.; Al-Haddad, K. On-board Microgrids for the More Electric Aircraft-Technology Review. IEEE Trans. Ind. Electron. 2019, 66, 5588-5599. [CrossRef]

2. De, D.; Castellazzi, A.; Lamantia, A. $1.2 \mathrm{~kW}$ dual-active bridge converter using SiC power MOSFETs and planar magnetics. In Proceedings of the 2014 International Power Electronics Conference (IPEC-Hiroshima 2014-ECCE ASIA), Hiroshima, Japan, 18-21 May 2014.

3. De, D.; Castellazzi, A.; Lopez-Arevalo, S.; Lamantia, A. SiC MOSFET based Avionic Power Supply. In Proceedings of the 7th IET International Conference on Power Electronics, Machines and Drives (PEMD 2014), Manchester, UK, 8-10 April 2014.

4. Giuliani, F.; Dipankar, D.; Delmonte, N.; Castellazzi, A.; Cova, P. Robust snubberless soft-switching power converter using SiC Power MOSFETs and bespoke thermal design. Microelectron. Reliab. 2014, 54, 1916-1920. [CrossRef]

5. Giuliani, F.; Delmonte, N.; Cova, P.; Costabeber, A.; Castellazzi, A. Soft-starting procedure for dual active bridge converter. In Proceedings of the 2015 IEEE 16th Workshop on Control and Modeling for Power Electronics (COMPEL), Vancouver, BC, Canada, 12-15 July 2015.

6. Castellazzi, A.; Gurpinar, E.; Wang, Z.; Hussein, A.; Garcia-Fernandez, P. Impact of Wide-Bandgap Technology on Renewable Energy and Smart-Grid Power Conversion Applications Including Storage. Energies 2019, 12, 4462. [CrossRef]

7. Krismer, F.; Kolar, J.W. Efficiency-Optimized High-Current Dual Active Bridge Converter for Automotive Applications. IEEE Trans. Ind. Electron. 2012, 59, 2745-2760. [CrossRef]

8. Maharana, S.; Mukherjee, S.; De, D.; Dash, A.; Castellazzi, A. Study of Dual Active Bridge with Modified Modulation Techniques for Reduced Link Current Peak Stress and Harmonics Losses in Magnetics. IEEE Trans. Ind. Appl. 2020, 56, 5035-5045. [CrossRef]

9. Higa, H.; Itoh, J. Zero voltage switching over entire load range and wide voltage variation of parallelly-connected dual-active-bridge converter using power-circulating operation. In Proceedings of the 2017 IEEE 3rd International Future Energy Electronics Conference and ECCE Asia (IFEEC 2017_ECCE Asia), Kaohsiung, Taiwan, 3-7 June 2017.

10. Nasirian, V.; Davoudi, A.; Lewis, F.; Guerrero, J.M. Distributed Adaptive Droop Control for DC Distribution Systems. IEEE Trans. Energy Convers. 2014, 29, 944-956. [CrossRef]

11. Rodrigues, W.A.; Oliveira, T.R.; Morais, L.M.F.; Rosa, A.H.R. Voltage and Power Balance Strategy without Communication for a Modular Solid State Transformer Based on Adaptive Droop Control. Energies 2018, 18, 1802. [CrossRef]

12. Sarlioglu, B.; Morris, C.T. More Electric Aircraft: Review, Challenges, and Opportunities for Commercial Transport Aircraft. IEEE Trans. Transp. Electrif. 2015, 1, 54-64. [CrossRef]

13. Wang, C.; Chen, J. Investigation on the Selection of Electric Power System Architecture for Future More Electric Aircraft. IEEE Trans. Transp. Electrif. 2018, 2, 563-576.

14. Wang, Z.; Castellazzi, A.; Saeed, S.; Navarro-Rodríguez, Á.; Garcia-Fernandez, P. Impact of SiC technology in a three-port active bridge converter for energy storage integrated solid state transformer applications. In Proceedings of the 2016 IEEE 4th Workshop on Wide Bandgap Power Devices and Applications (WiPDA), Fayetteville, AR, USA, 7-9 November 2016.

15. Wang, Z.; Castellazzi, A. Device loss model of a fully SiC based dual active bridge considering the effect of synchronous rectification and deadtime. In Proceedings of the 2017 IEEE Southern Power Electronics Conference (SPEC), Puerto Varas, Chile, 4-7 December 2017.

16. Castellazzi, A.; Fayyaz, A.; Gurpinar, E.; Hussein, A.; Li, J.; Mouawad, B. Multi-Chip SiC MOSFET Power Modules for Standard Manufacturing, Mounting and Cooling. In Proceedings of the 2018 International Power Electronics Conference (IPEC-Niigata 2018_ECCE Asia), Niigata, Japan, 20-24 May 2018.

Publisher's Note: MDPI stays neutral with regard to jurisdictional claims in published maps and institutional affiliations. 